\title{
Role of Glycolic and Salicylic Acid Chemical Peel in the Management of Post-acne Pigmentation: A Literature Review
}

\author{
Bharathi Ravikumar ${ }^{1}$, Indra Devi ${ }^{2}$
}

\begin{abstract}
Chemical peeling is a process wherein one or more chemical agents are applied to the skin. It causes partial destruction of the epidermis or dermis with exfoliation or desquamation followed by resurfacing of the epidermis and remodeling of the collagen and elastic fibers. It is a type of controlled cosmetic chemical burn which is one of the treatments for acne and post-acne pigmentation. Post-acne hyperpigmentation (PAH) is very common in acne patients which can occur in all skin types, although it is more common in dark skin types which affects both men and women equally. Many studies have been conducted for post-acne pigmentation by combining chemical peeling with lasers or with topical medications. Only a few studies have reported the efficacy of chemical peeling alone in the treatment of post-acne pigmentation. Thus, this review focuses on the efficacy of glycolic and salicylic acid chemical peels used in the management of post-inflammatory pigmentation. Keywords: Chemical peeling, Glycolic acid, Post-acne hyperpigmentation, Salicylic acid.

Annals of SBV (2021): 10.5005/jp-journals-10085-8132
\end{abstract}

\section{INTRODUCTION}

Post-acne pigmentation is a frequently encountered challenge in dermatological practice. Reports indicate that post-acne pigmentation is of widespread concern in the Asian population with patients often indicating that the pigmentation problems are as much, if not more distressing than the original acne lesions. ${ }^{1}$ Although it may be a common perception that it is easy to diagnose post-acne pigmentation, there are significant variations amongst researchers in assessing its presence. ${ }^{2}$ The Asian Acne Board (AAB), a component of the Global Alliance to Improve Outcomes in Acne, is an international group of dermatologists with an interest in advancing knowledge of acne and disseminating better clinical practices that may improve patient outcomes. In recent meetings, the $A A B$ has sought to address ways in which to improve assessment and management of post-acne pigmentation, which is a prevalent problem in the Asian population. ${ }^{3}$

Chemical peeling is a popular, relatively inexpensive modality that has shown to be an effective therapeutic option in the treatment of active acne lesions, post-acne hyperpigmentation $(\mathrm{PAH})$, and to rejuvenate skin. Widely different types of peels are available with different mechanisms of action, which can be modulated by altering concentrations. ${ }^{4} \mathrm{~A}$ literature search is done using the keywords chemical peeling, post-inflammatory hyperpigmentation, glycolic acid (GA), and salicylic acid (SA) in databases such as PubMed and Scopus which revealed that 24 articles out of which 10 relevant full-text articles published in the time period between 2010 and 2020 were reviewed.

\section{Post-inflammatory Hyperpigmentation}

Post-inflammatory hyperpigmentation $(\mathrm{PIH})$ is a medical term that refers to the discoloration of the skin after an inflammatory wound. This is the skin's natural response to inflammation. Depending on the skin type and the depth of discoloration, $\mathrm{PIH}$ presents flat discoloration areas on the skin (macro), ranging from pink to red, purple, brown, or black. Post-inflammatory hyperpigmentation is characterized by an acquired increase in skin pigmentation
1,2Department of DVL, Mahatma Gandhi Medical College and Research Institute, Puducherry, India

Corresponding Author: Bharathi Ravikumar, Department of DVL, Mahatma Gandhi Medical College and Research Institute, Puducherry, India, Phone: +91 8754499788, e-mail: dr.bharathi09@gmail.com

How to cite this article: Ravikumar B, Devi I. Role of Glycolic and Salicylic Acid Chemical Peel in the Management of Post-acne Pigmentation: A Literature Review. Ann SBV 2021;10(1):8-11.

Source of support: Nil

Conflict of interest: None

secondary to the inflammatory process. Excessive pigmentation may occur in the epidermis or the epidermis and dermis. Pigmentation after inflammation may be sequelae of diseases such as acne. ${ }^{5}$ Post-inflammatory hyperpigmentation is caused by one of two mechanisms, leading to epidermal melanosis or dermal myelopathy. The inflammatory response of the epidermis leads to the release of arachidonic acid and subsequent oxidation to prostaglandins, leukotrienes, and other products.

This increased stimulation and transfer of melanin particles lead to excessive melanin degeneration of the epidermis. Conversely, when inflammation destroys the basal cell layer, causing the melanin to be released and subsequently captured by macrophages in the papillary dermis, skin melanosis occurs, which is also called pigmented incontinence. ${ }^{6}$

With acne, papules, and pustules, the infection may spread to the deep layer of skin called the dermis. The infected area produces more melanin than the normal area, resulting in abnormal darkness. Therefore, infection of hair follicles and sebaceous glands is the real cause of hyperpigmentation. In most cases, if the acne is not severe, it will not leave pigmentation. Squeezing and popping acne can also cause pigmentation. Exposure to the sun is the main cause of acne and pigmentation. Melanocytes are activated by sunlight (ultraviolet rays) to produce excessive melanin. Even without treatment, PIH will gradually disappear over time. It may take 3-24 months for PIH to completely disappear, although in some cases

(c) The Author(s). 2021 Open Access This article is distributed under the terms of the Creative Commons Attribution 4.0 International License (https:// creativecommons.org/licenses/by-nc/4.0/), which permits unrestricted use, distribution, and non-commercial reproduction in any medium, provided you give appropriate credit to the original author(s) and the source, provide a link to the Creative Commons license, and indicate if changes were made. The Creative Commons Public Domain Dedication waiver (http://creativecommons.org/publicdomain/zero/1.0/) applies to the data made available in this article, unless otherwise stated. 
it may take longer. The greater the contrast between the macula and natural skin tone, the longer it takes to fade.

\section{Indian Scenario}

In India, most subjects with a history of acne show pigmentation after inflammation:

Seventy percent of men and women suffer from this disease before the age of 35 . With age, this prevalence rate drops rapidly, involving $<10 \%$ of people over 50 . This observation is directly related to the high incidence of acne observed in the study. ${ }^{7}$ Post-inflammatory hyperpigmentation may last for several months to several years and may severely impair the quality of life of affected individuals. Compared with short-term acute inflammation, the severity of PIH is higher in long-term and/or recurrent inflammation. Epidermal inflammation can cause the production and release of various cytokines, prostaglandins, and leukotrienes, thereby stimulating the epidermis. Melanocytes lead to increased synthesis of total melanin. Most of these factors are also produced under solar stimulation, supporting the role of UV exposure in the initiation and deterioration of $\mathrm{PIH}$. In addition, skin inflammation also causes damage to the basal layer, leading to the leakage of melanin from the basal keratinocytes and subsequent accumulation of melanocytes in the dermis, thereby exacerbating skin hyperpigmentation.

\section{Chemical Peeling}

Interventional therapies for treating skin pigmentation after acne are focused on obtaining the best results. Therefore, the aggressive approach is replaced by a less aggressive and less safe program that can minimize the complications and optimize the results. Chemical peels have been found to reduce post-acne rosacea and reduce the pigmentation of the healed lesions and scars. Also besides, the chemical peel improves the depth, contour, and helps in softening of related scars through its effect on collagen remodeling and stimulation of new collagen activity. Most importantly, the peel can also improve the texture and gloss. ${ }^{8}$ Therefore, before seeking the use of laser and light for surface treatment, chemical peels form a bridging link that can create an interventional primer to treat pigmented acne marks in the skin tone.

In dark skin prototypes 4-6, thick stratum corneum, large melanosomes, thick dermis, and abundant fibroblasts constitute the morphological characteristics. Chemical peel promotes improvement by thinning the stratum corneum and regenerating a dense epidermis, which evenly reflects light across the skin surface, and imparts texture improvement and lightening effects while eliminating epidermal melanin and preventing melanin from transferring to keratinocytes.

\section{Superficial Peeling Agents}

\section{Alpha-hydroxy Acids}

Alpha-hydroxy acids (AHA) constitute a cluster of carboxylic acids with a hydroxyl group attached to the alpha position of the carboxyl group. They are derived from fruits [such as apples (malic acid), grapes (tartaric acid), lemons and oranges (citric acid), sugar cane (GA), and milk (lactic acid)]. ${ }^{9}$ At lower concentrations, AHA can cause keratinocytes to stick to each other whereas, at higher concentrations, AHA will promote the decomposition of the epidermis. Alpha-hydroxy acids need to be neutralized to stop its effect. This can be achieved by water, sodium bicarbonate, sodium hydroxide, or ammonium salt solution. Alpha-hydroxy acids do not cause frosting. Glycolic acid is the most common AHA peeling agent. It is available as an esterified, buffered, partially neutralized, or free acid solution. ${ }^{10}$ It also has a pKa of 3.83 and is soluble in water which is an important concept in chemical peeling. The lower the pKa value, the higher the utilization rate of free acid and the stronger the peeling force.

\section{Beta-hydroxy Acids}

Salicylic acid: Salicylic acid (also known as ortho-hydroxybenzoic acid) is a naturally occurring beta-hydroxy acid derived from sweet birch, holly leaves, and willow bark. It has a pKa of 2.97 and is hardly soluble in water. It destroys the intercellular lipids that are covalently attached to the keratinized envelope surrounding the keratinized keratinocytes. ${ }^{11}$ This leads to the peeling of the stratum corneum and activation of basal keratinocytes and fibroblasts. Salicylic acid has mild analgesic, antimicrobial, stratum corneum breakdown, and anti-inflammatory effects. For surface chemical peeling, the concentration of SA in ethanol or polyethylene glycol ethanol is $10-30 \%$. It is used on the skin for 3-5 minutes and causes a brief burning sensation before its analgesic effect prevails. The evaporation of the hydroethanol base will leave a white precipitate, which is usually mistaken for frost. It is important to note that SA peels do not cause frosting or need to be neutralized. If they are applied to a large surface at once, they theoretically also have the risk of salicylism. Salicylic acid peel is particularly suitable for acne and inflammatory acne as well as oily skin.

Lipohydroxy acid: C8-aliphatic hydroxy acid (also known as octanoyl salicylic acid, 2-hydroxy-5-octanoyl benzoic acid, or aliphatic-hydroxy hydroxy acid) is a lipophilic derivative of SA. The acyl fatty chain forms the carbon atoms of the benzene ring. Lipid hydroxy acids target the keratinocyte structure to uniformly separate keratinocytes. The increased lipophilicity of lipohydroxy acid is that when compared with SA, its mechanism of action in the epidermis and the sebaceous sac is more targeted, and it has a greater keratolytic effect. The concentration of fatty hydroxy acid is $5-10 \%$, and neutralization is not required. It also has antibacterial, anti-inflammatory, and non-comedogenic properties. ${ }^{4}$

Jessner solution: The Jessner solution (JS) is composed of SA (14 g), resorcinol (14 g), lactic acid 85\% (14 g), and ethanol (100 mL qs). This is a clear amber solution that needs to be kept brown to avoid photooxidation. Jessner solution usually only appears after two or three coats of minimal erythema and delicate spot-like frosting and it does not need to be neutralized. Jessner solution can be used as a preparative peeling agent and also enhance the permeability of another stripping agent (such as TCA).

Pyruvic acid: Pyruvate, also known as acetoformic acid, is a keto acid different from AHA. It has a carbonyl group at the position of the carboxyl group. It is an effective stripping agent with a pKa of 2.39 and is soluble in water and ethanol. Pyruvate is physiologically converted into lactic acid, and the concentration is 40 to $70 \%$. It causes ablation of the stratum corneum and separation of the dermis-epidermis, resulting in a reduction in the thickness of the epidermis. In long-term, it induces an increase in the deposition of collagen, elastin, and glycoprotein in the papillary dermis. Pyruvate can cause severe pain during use, and its vapor can be irritating.

Resorcinol: Resorcinol is a phenol derivative, which has a pKa of 9.32 and is soluble in water, ether, and ethanol. Resorcinol stimulates 
the formation of prostaglandin E2 and breaks the hydrogen bond of keratin, which shows that it has keratolytic and bactericidal properties. Resorcinol has a concentration of $10-50 \%$ and is used for chemical peeling causing frosting. It is used to treat acne, seborrheic dermatitis, and melasma. ${ }^{12}$ Adverse reactions related to resorcinol include allergic and irritant contact dermatitis, mucinous edema, chronic allergy, and hemoglobinemia caused by long-term use.

Clinicians and patients often use chemical peels as an adjunct to drug treatment in acne because they can produce complementary rapid treatment effects and improve skin appearance and texture. The main effect may be to treat acne while reducing inflammatory lesions. Peel can make the topical acne agent penetrate the skin more effectively, and can improve PIH. Although the 2009 American Academy of Dermatology guidelines point out that more evidence is needed to determine best practices, ${ }^{13}$ clinical experience shows broad application prospects. Researched peels that can treat acne include SA, GA, LHA, and JSs.

Trichloroacetic acid peels: In various cases, such as PIH, melanoma, and friction skin melanosis, TCA peeling is required. Seventy percent of GA followed by 35\% TCA peeling, in conditions such as $\mathrm{PIH}, \mathrm{PAH}$, melasma, and other pigmented chromatic aberrations, produces a deeper and more uniform peeling than TCA alone. Another study by Sachdeva ${ }^{14}$ showed that the efficacy of $10-20 \%$ of GA peel and $35-70 \%$ of TCA in PIH showed that GA peel is safer and more effective than TCA peel, although the difference is not statistically significant.

The study of Sacchidanand et al. ${ }^{15}$ compared the effects of $15 \%$ TCA peel and 50\% GA peel in frictional melanosis and found that both peeling agents are equally effective. However, the response of TCA was better than that of GA peel, and the difference in response was not statistically significant. Compared to GA peeling, TCA peeling has more visible erythema and burning sensation. In addition, TCA peeling is related to the icing reaction, whereas in GA peeling is completely absent. Hence, it should be noted that without proper supervision and sun protection, there are more likely chances of PIH to worsen by using TCA peel.

Glycolic acid peel: Glycolic acid peel is the smallest alpha-hydroxy acid, so it easily penetrates the skin with a concentration range is $20-70 \%$. Glycolic peel with a percentage of $50-70 \%$ applied for 2-5 minutes is classified as a shallow layer, and 70\% GA applied for 3-15 minutes is classified as a medium-deep layer. The possible mechanism of pigmentation in patients with PIH may be due to the dissolution of the pigmented skin epidermis, the re-epithelialization of the new pigmented skin, and the new pigmented epidermis covering the dermis. In addition, since it has a structure similar to ascorbic acid, it may have a direct depigmentation effect on the skin. Avoiding sunlight to break the stratum corneum is very important for the skin-lightening effect. Sharad ${ }^{10}$ believes that GA peels can be used for post-acne pigmentation of Fitzpatrick III and IV skin types in India. In addition, she recommends GA peeling for 15 consecutive days, i.e., $20 \%$, followed by $35 \%$, peeling 8 to 10 times every 15 days, which is related to the removal of the lesion without any adverse effects. Burns et al. ${ }^{16}$ conducted a preliminary study where he performed a series of GA peels on PIH to skin types IV to $\mathrm{VI}$ and has been proven to be effective in treating dark skin patients without adverse effects. This is further confirmed by another study conducted by Grover and Reddu, ${ }^{17}$ where PIH and scar formation both showed excellent improvement in type III to V skin, and the overall skin texture was improved. They also commented that the improvement of PIH in patients secondary to superficial burns or medical treatment was poor.

Comparison of GA peels with other methods: In the study of Garg et al., ${ }^{18}$ the efficacy and tolerance of $35 \%$ GA peel and $20 \%$ SA-10\% mandelic acid (SMP) peel in active acne were compared. Although SMP had better results on post-acne scars, active acne, and postacne pigmentation at the end of the study, 35\% GA and SMP both showed comparable and significant improvement in PIH after acne.

In addition, the latest study by Sarkar et al. ${ }^{19}$ compared the safety and effectiveness of GA peel in combination with a modified Kligman formula (MKF), which contains $2 \%$ hydroquinone, $0.05 \%$ vitamin D formic acid, and hydrogenated Cortisone 1, where using GA with MKF showed a better improvement than MKF alone. However, side effects like erythema, burning, and desquamation were observed in the peel group.

SA peel: It has been proven that SA peeling combined with $4 \%$ hydroquinone or used alone can safely and effectively reduce $\mathrm{PIH}$ in patients with dark skin (especially SPT IV and V). Although SA peeling is often used in combination with hydroquinone, there is a lack of literary studies to incorporate it into the routine patient treatment protocol. In 2009, Joshi et al. ${ }^{20}$ conducted a study to evaluate the efficacy and safety of SA peeling on 10 patients with $\mathrm{PIH}$ types IV to VI. These patients received continuous SA peeling on half of their faces (20-30\%) and the other half has no treatment. The authors concluded that there was a nominal clinical improvement in $\mathrm{PIH}$ on the treatment side compared with the control group, but the clinical study did not show a statistically significant improvement. Grimes ${ }^{21}$ and Bari et al. ${ }^{22}$ conducted similar studies to treat various facial skin diseases including PIH. Grimes used hydroquinone series SA peeling and later used $30 \%$ SA peeling alone. Both studies showed encouraging results. Ahn and $\mathrm{Kim}^{23}$ also reported that patients with acne-induced $\mathrm{PIH}$ with $30 \%$ SA peel have lightening with thereby concomitant reduction of erythema, dryness, and scaling. While choosing chemical peels for dark-skinned races, this lightening effect can be very fascinating.

Comparison of SA peel with other methods: In a study, 20 to $30 \%$ of SA peel was compared with $0.1 \%$ topical retinoic acid solution between three groups. In the first and second groups, SA peel and retinoid were used alone, respectively, whereas both were used in the third group. Compared to groups I and II, the combination therapy (third group) had a significantly better effect. ${ }^{24}$ In addition, the combination therapy is well tolerated, has fewer adverse reactions, and has a low recurrence rate.

\section{Conclusion}

From the review, it is apparent that both glycolic and SA chemical peel were found to be effective in the treatment of post-acne pigmentation. Chemical peeling is a quick and inexpensive procedure that provides a controlled treatment and also gives the patients a safe and alternate treatment option. It discourages the patients to use the counter fairness or depigmenting creams and also overcomes the abuse of hydroquinone and steroid preparations which have their own array of side effects.

\section{References}

1. Kircik LH. Efficacy and safety of azelaic acid (AzA) gel $15 \%$ in the treatment of post-inflammatory hyperpigmentation and acne: a 
16-week, baseline-controlled study. J Drugs Dermatol 2011;10(6):586590.

2. Matarasso SL, Glogau RG, Markey AC. Wood's lamp for super-ficial chemical peels. J Am Acad Dermatol 1994;30(6):988-992. DOI: 10.1016/s0190-9622(94)70124-5.

3. Woolery-Lloyd H, Kammer JN. Treatment of hyperpigmentation. Semin Cutan Med Surg 2011;30(3):171-175. DOI: 10.1016/j.sder.2011.06.004.

4. Rendon MI, Berson DS, Cohen JL, Roberts WE, Starker I, Wang B. Evidence and considerations in the application of chemical peels in skin disorders and aesthetic resurfacing. J Clin Aesthet Dermatol 2010;3(7):32.

5. Davis EC, Callender VD. Postinflammatory hyperpigmentation: a review of the epidemiology, clinical features, and treatment options in skin of color. J Clin Aesthet Dermatol 2010;3(7):20-31.

6. Akamutsu H, Horio T, Hattori K. Increased hydrogen peroxide generation by neutrophils from patients with acne inflammation. Int J Dermatol 2003;42(5):366-369. DOI: 10.1046/j.1365-4362.2003.01540.x.

7. Hourblin V, Nouveau S, Roy N, de Lacharrière O. Skin complexion and pigmentary disorders in facial skin of 1204 women in 4 Indian cities. Indian J Dermatol Venereol Leprol 2014;80(5):395-401. DOI: 10.4103/0378-6323.140290.

8. Grimes PE. Management of hyperpigmentation in darker racial ethnic groups. Semin Cutan Med Surg 2009;28(2):77-85. DOI: 10.1016/j. sder.2009.04.001.

9. Fabbrocini GDP, De Padova MP, Tosti A. Chemical peels:what's new and what isn't new but still works well. Facial Plast Surg 2009;25(5):329-336. DOI: 10.1055/s-0029-1243082.

10. Sharad J. Glycolic acid peel therapy - a current review. Clin Cosmet Investig Dermatol 2013;6:281-288. DOI: 10.2147/CCID.S34029.

11. Zakopoulou N, Kontochristopoulos G. Superficial chemical peels. J Cosmet Dermatol 2006;5(3):246-253. DOI: 10.1111/j.14732165.2006.00254.x.

12. Boer J, Jemec GB. Resorcinol peels as a possible self-treatmentof painful nodules in hidradenitis suppurativa. Clin Exp Dermatol 2010;35(1):36-40. DOI: 10.1111/j.1365-2230.2009.03377.x.

13. Strauss JS, Krowchuk DP, Leyden JJ, et al. Guidelines of care for acne vulgaris management. J Am Acad Dermatol 2007;56(4):651-663. DOI: 10.1016/j.jaad.2006.08.048.

14. Sachdeva S. Comparative efficacy of $10-20 \%$ trichloroacetic acid and $35-70 \%$ glycolic acid peel in 60 cases of melasma, freckles, lentigenes and post inflammatory hperpigmentation. J Pak Assoc Derma 2006;16:76-80.

15. Sacchidanand S, Shetty $A B$, Leelavathy B. Efficacy of $15 \%$ trichloroacetic acid and $50 \%$ glycolic acid peel in the treatment of frictional melanosis: a comparative study. J Cutan Aesthet Surg 2015;8(1):37-41. DOI: 10.4103/0974-2077.155078.

16. Burns RL, Prevost-Blank PL, Laury MA, Laury TB, Faira DT, Fiverson AP. Glycolic acid peels for post-inflammatory hyperpigmenatation in black patients: a comparative study. Dermatol Surg 1997;23(3):171174. DOI: 10.1111/j.1524-4725.1997.tb00014.x.

17. Grover C, Reddu BS. The therapeutic value of glycolic acid peels in dermatology. Indian J Dermatol Venereol Leprol 2003;69(2):148-150.

18. Garg VK, Sinha S, Sarkar R. Glycolic acid peels versus salicylicmandelic acid peels in active acne vulgaris and post-acne scarring and hyperpigmentation: a comparative study. Dermatol Surg 2009;35(1):59-65. DOI: 10.1111/j.1524-4725.2008.34383.x.

19. Sarkar R, Parmar NV, Kapoor S. Treatment of postinflammatory hyperpigmentation with a combination of glycolic acid peels and a topical regimen in dark-skinned patients: a comparative study. Dermatol Surg 2017;43(4):566-573. DOI: 10.1097/ DSS.0000000000001007.

20. Joshi SS, Boone SL, Alam M, Yoo S, White L, Rademaker A, et al. Effectiveness, safety, and effect on quality of life of topical salicylic acid peels for treatment of postinflammatory hyperpigmentation in dark skin. Dermatol Surg 2009;35(4):638-644. DOI: 10.1111/j.15244725.2009.01103.x.

21. Grimes PE. The safety and efficacy of salicylic acid chemical peel in darker racial-ethnic groups. Dermatol Surg 1999;25(1):18-22. DOI: 10.1046/j.1524-4725.1999.08145.x.

22. Bari AU, Iqbal Z, Rahman SB. Tolerance and safety of superficial chemical peeling with salicyclic acid in various facial dermatoses. Indian J Dermatol Venereol Leprol 2005;71(2):87-90. DOI: 10.4103/0378-6323.13990.

23. Ahn HH, Kim IH. Whitening effect of salicyclic acid peels in Asian patients. Dermatol Surg 2006;32(3):372-375. DOI: 10.1111/j.15244725.2006.32075.x.

24. Ali BMM, Gheida SF, Mahdy NAE, Sadek SN. Evaluation of salicylic acid peeling in comparison with topical tretinoin in the treatment of postinflammatory hyperpigmentation. J Cosmet Dermatol 2016;16:52-56. 